\title{
DUALITY BETWEEN LOGICS AND EQUIVALENCE RELATIONS
}

BY

\author{
DANIELE MUNDICI
}

\begin{abstract}
Assuming $\omega$ is the only measurable cardinal, we prove:
(i) Let $\sim$ be an equivalence relation such that $\sim=\equiv_{L}$ for some logic $L \leqslant L^{*}$ satisfying Robinson's consistency theorem (with $L^{*}$ arbitrary); then there exists a strongest logic $L^{+} \leqslant L^{*}$ such that $\sim=\equiv_{L^{+}}$; in addition, $L^{+}$is countably compact if $\sim \neq \cong$.

(ii) Let $\dot{\sim}$ be an equivalence relation such that $\sim=\equiv_{L^{0}}$ for some logic $L^{0}$ satisfying Robinson's consistency theorem and whose sentences of any type $\tau$ are (up to equivalence) equinumerous with some cardinal $\kappa_{\tau}$; then $L^{0}$ is the unique logic $L$ such that $\sim=\equiv_{L}$; furthermore, $L^{0}$ is compact and obeys Craig's interpolation theorem.

We finally give an algebraic characterization of those equivalence relations which are equal to $\equiv_{L}$ for some compact logic $L$ obeying Craig's interpolation theorem and whose sentences are equinumerous with some cardinal.
\end{abstract}

0. Introduction. This paper is concerned with the following problem: what can we say about the inverse of the map taking logic $L$ into $L$-elementary equivalence $\equiv_{L}$ ? We shall derive invertibility results in case $L$ satisfies Robinson's consistency theorem. In studying the interrelations between logics and equivalence relations on structures, the notion of Robinson's consistency has many applications (see [Mu2]): on one hand, any logic $L$ in which $\operatorname{Stc}_{L}(\tau)$ is a set satisfies Robinson's consistency theorem iff $L$ is compact and satisfies Craig's interpolation theorem, by a result due to the present author and, independently, to Makowsky and Shelah (see [Mu3] and [MS1]); on the other hand, Robinson's consistency only depends on $\equiv_{L}$ rather than on $L$, and $\equiv_{L}$ has a simpler structure: in fact, Robinson's consistency has a very neat algebraic characterization in terms of amalgamation and joint embedding properties (see [Mu3] and [Mu6]); furthermore, one can relativize this notion to equivalence relations on smaller classes of structures: thus, for instance, in [Mu1] it is proved that on the class of countable structures of finite type there are just two nonpathological equivalence relations satisfying Robinson's consistency, namely $\cong$ and $\equiv ;$ in the light of the above-mentioned equivalence "Robinson $=$ Craig + Compactness", this might be also regarded as a partial answer to H. Friedman's fourth problem in $[\mathbf{F r}]$ of finding proper extensions of first-order logic still satisfying compactness and interpolation. Concerning Friedman's third problem, too, the techniques developed in [Mu5] for the study of Robinson's consistency in infinitary logics yield such results as "no logic $L$ strictly between $L_{\infty \omega}$ and $L_{\infty \infty}$ obeys Craig's

Received by the editors August 4, 1980.

1980 Mathematics Subject Classification. Primary 03C95; Secondary 03C30, 03C75, 03C40, 03C55, 03 E55. 
interpolation, or Robinson's consistency, unless $\equiv_{L}=\cong$ (in which case Friedman's problem is still open)".

With the exception of the result in [Mu1], all the above-mentioned results depend on such special set-theoretical hypotheses as $\neg 0^{\#}$ or $\neg L^{\mu}$ (there is no inner model with an uncountable measurable cardinal); incidentally, this gives an idea of the sensitivity of soft model theory for pluralism in set theory. Also the main results of this paper depend (as far as the author can see) on the following assumption: $\omega$ is the only measurable cardinal.

Hoping that the above discussion has convinced some readers that assuming Robinson's consistency is not an arbitrary restriction, if $\sim$ is an equivalence relation on the class of all structures, we say that $\sim$ is representable iff $\sim=\equiv_{L}$, for some $\operatorname{logic} L ; \equiv_{L}$ satisfies Robinson's consistency theorem iff $L$ does; this is faithfully generalized to the case of an arbitrary equivalence relation $\sim$, called for short a Robinson equivalence relation. (See Definition 1.1.)

Our first main result (Theorem 3.1) states that for any representable Robinson equivalence relation $\sim=\equiv_{L}$ with $L \leqslant L^{*}$ and $L^{*}$ arbitrary, there is a strongest logic $L^{+} \leqslant L^{*}$ such that $\equiv_{L^{+}}=\sim$; in addition, $L^{+}$(hence any weaker logic than $L^{+}$still representing $\sim$ ) is countably compact if $\cong \neq \sim$.

Thus, although there is no bijection between representable equivalence relations and logics, under the Robinson assumption, a bijection exists if one limits oneself to the strongest logic $\leqslant L^{*}$ representing $\sim$; notice that the mere existence of a strongest logic is not a trivial fact, in that our notion of a logic incorporates from the very start such features as closure under first-order operations, among which is relativization.

If one considers the case of bounded equivalence relations, i.e. when the equivalence classes of type $\tau$ form a class having a cardinality (this is the case, e.g., $\sim=\equiv_{L}$, for a $\operatorname{logic} L$ in which $\operatorname{Stc}_{L}(\tau)$ is a set) then the inversion problem is completely solved. As a matter of fact, our second main result (Theorem 4.2) states that any bounded representable Robinson equivalence relation $\sim$ is uniquely represented; also, the unique logic $L$ such that $\equiv_{L}=\sim$ is compact and obeys Craig's interpolation.

Thus the function $L \mapsto \equiv_{L}$ maps logics satisfying compactness and Craig's interpolation one-one onto representable Robinson equivalence relations (under the abovementioned "boundedness" restriction).

In Corollary 4.4 we prove that only one logic represents elementary equivalence, namely first-order logic (notice that if one only considers representations by compact logics, then the above corollary easily follows from Lindström's theorem (see [Li] or [FI]), or even from a "finite cover" argument).

Having solved the uniqueness problem we take up in $\$ 5$ the problem of the existence of representations of $\sim$ as $\equiv_{L}$ for $L$ a logic satisfying compactness and Craig's interpolation. For $X$ a union of equivalence classes of $\sim$, we let $\operatorname{span}(X)$ be the collection of those classes $Y$ which can be obtained from $X$ by repeated applications of the first-order operations; we also let $X \in \operatorname{hull}(\sim)$ iff for all $Y \in \operatorname{span}(X), Y$ is still a union of equivalence classes of $\sim$; we call $\sim$ separable iff 
whenever not $\mathfrak{A} \sim \mathscr{B}$ there is $X \in \operatorname{hull}(\sim)$ separating $\mathfrak{A}$ and $\mathfrak{B}$; we prove that (if $\omega$ is the only measurable cardinal) the following are equivalent: (i) $\sim$ is bounded, separable, Robinson, preserved under reduct, finer than $\equiv$ and coarser than $\cong$, and (ii) $\sim=\equiv_{L}$ for precisely one logic $L$; in addition, $L$ is compact, obeys interpolation, $\left|\operatorname{Stc}_{L} \tau\right|$ exists, and each sentence in $L$ is of finite type.

The above representation theorem allows one to give an equivalent algebraic reformulation of Friedman's fourth problem in [Fr]. The author is aware that one of the possible answers to this problem would make his results of $\S \S 4$ and 5 only applicable to first-order logic and elementary equivalence. Still, he has decided to include these results here, hoping that either (i): Friedman's fourth problem has no such answer, or (ii): if $\neg$ (i) holds, still the techniques used in $\S \S 4-6$ may be of some help to obtain $\neg$ (i) itself, or, at least, (iii): no fact implying both $\neg$ (i) and $\neg$ (ii) occurs before July 14, 1989.

1. Preliminaries. Throughout this paper $\alpha, \beta, \ldots$ denote ordinals and $\kappa, \lambda, \mu, \nu, \ldots$ denote infinite cardinals; $\tau, \tau^{\prime}, \tau^{\prime \prime}$ denote sets of sorts and symbols, called types; $\mathfrak{A}$, $\mathfrak{B}, \mathfrak{D}, \mathfrak{M}, \mathfrak{R}$ denote (many-sorted) structures, whose universes $A, B, D, M$ and $N$ are always understood to be sets; $\operatorname{Str}(\tau)$ is the class of all structures of type $\tau$ (compare with [Fe]); following [MSS], we let $I \mathfrak{A}=\{\mathfrak{B} \mid \mathfrak{B} \cong \mathfrak{A}\}$. A name-changer $\rho: \tau \rightarrow \tau^{\prime}$ is a one-one function from $\tau$ onto $\tau^{\prime}$ taking relation (resp., function) symbols into relation (resp., function) symbols of the same arity; for $\mathfrak{A} \in \operatorname{Str}(\tau), \mathfrak{A}^{\rho}$ is the structure in which each symbol $S \in \tau^{\prime}$ is interpreted just as $\rho^{-1}(S)$ is interpreted in $\mathfrak{A}$. We also let $\tau(\mathfrak{A})$ be the type of $\mathfrak{A}$.

A logic $L$ is an ordered pair $\left(\operatorname{Stc}_{L}, \xi_{L}\right)$ satisfying the basic axioms of occurrence [Ba], expansion, renaming, isomorphism and which is closed under the familiar operations of negation, conjunction, quantification, and contains the atomic sentences as in [Fe, pp. 155-157]; formulas are sentences with additional constant symbols, as in [Fe, p. 156], and relativization of formula $\varphi$ to formula $\psi(x)$ is always allowed in our logics, where $\psi(x)$ is a boolean combination of atomic formulas, and gives formula $\varphi^{\{x \mid \psi(x)\}}$, whose semantics is the familiar one, as explained in [FI]. See also $\S 5$.

Notice that all structures and all types are admitted in $L$, that types need not be finite, that $\operatorname{Stc}_{L}(\tau)$ need not be a set, that in the sentences of $L$ arbitrarily large sets of symbols may occur; if $\varphi \in \operatorname{Stc}_{L}(\tau)$, then we merely say that $\varphi$ is of type $\tau$.

For $\mathfrak{M} \in \operatorname{Str}(\tau)$,

$$
\operatorname{th}_{L} \mathfrak{M}=\left\{\varphi \in \operatorname{Stc}_{L}(\tau) \mid \mathfrak{M F}_{L} \varphi\right\}
$$

for $T \subseteq \operatorname{Stc}_{L}(\tau)$,

$$
\bmod _{L} T=\left\{\mathfrak{M} \in \operatorname{Str}(\tau) \mid \mathfrak{M}_{L} T\right\} .
$$

$\mathfrak{U} \equiv_{L} \mathfrak{B}$ means that $\operatorname{th}_{L} \mathfrak{A}=\operatorname{th}_{L} \mathfrak{B} . K$ is an elementary class in $L$ of type $\tau$ iff ( $K \subseteq \operatorname{Str}(\tau)$ and) $K=\bmod _{L} \varphi$ for some $\varphi \in \operatorname{Stc}_{L}(\tau)$; a structure $\mathfrak{A}$ is characterizable in $L$ by its theory iff $I \mathfrak{A}=\bmod _{L} \mathrm{th}_{L} \mathfrak{A}$, i.e. iff the structures which are $L$-elementarily equivalent to $\mathfrak{A}$ are precisely those which are isomorphic to $\mathfrak{A}$. $\equiv$ is elementary equivalence. 
An equivalence relation $\sim$ is representable iff $\sim=\equiv_{L}$ for some logic $L$; given logics $L^{\prime}$ and $L^{\prime \prime}$ we write $L^{\prime} \leqslant L^{\prime \prime}$ iff for each type $\tau$ and $\varphi^{\prime} \in \operatorname{Stc}_{L^{\prime}}(\tau)$ there exists $\varphi^{\prime \prime} \in \operatorname{Stc}_{L^{\prime \prime}}(\tau)$ such that $\bmod _{L^{\prime}} \varphi^{\prime}=\bmod _{L^{\prime \prime}} \varphi^{\prime \prime}$. If $L^{\prime} \leqslant L^{\prime \prime}$ and $L^{\prime \prime} \leqslant L^{\prime}$ then we simply write $L^{\prime}=L^{\prime \prime}$ : as a matter of fact, in this case $L^{\prime}$ and $L^{\prime \prime}$ have the same expressive power (i.e. the same elementary classes) even if they might differ in the way an elementary class is represented by sentences. When $L^{\prime} \leqslant L^{\prime \prime}$ we also say that $L^{\prime \prime}$ is stronger than $L^{\prime}$.

We assume the reader is familiar with compact and countably compact logics and with logics obeying Craig's interpolation theorem (see [MS] or [MSS]). Generalizing a definition in [MS] we say that logic $L$ is $\kappa$-relatively compact ( $\kappa$-r.c.) iff for any type $\tau$ and classes of sentences $\Sigma$ and $\Gamma$ of type $\tau$ with $|\Sigma|=\kappa$, if for each $\Sigma^{\prime} \subseteq \Sigma$ with $\left|\Sigma^{\prime}\right|<\kappa, \Gamma \cup \Sigma^{\prime}$ has a model, then $\Gamma \cup \Sigma$ has a model.

The familiar notion of logic $L$ satisfying Robinson's consistency theorem (see [Mu1-Mu6]) is faithfully transposed to the case of an equivalence relation via the following stipulation:

1.1 Definition. Let $\sim$ be an equivalence relation on the class of all structures; then $\sim$ has the Robinson property (or, $\sim$ is a Robinson equivalence relation) iff $\forall \mathfrak{M} \in \operatorname{Str}\left(\tau^{\prime}\right), \quad \forall \mathfrak{N} \in \operatorname{Str}\left(\tau^{\prime \prime}\right), \quad$ if $\tau=\tau^{\prime} \cap \tau^{\prime \prime}$ and $\mathfrak{M} \uparrow \tau \sim \mathfrak{N} \uparrow \tau$, then $\exists \mathfrak{D} \in$ $\operatorname{Str}\left(\tau^{\prime} \cup \tau^{\prime \prime}\right)$ such that $\mathscr{D} \mid \tau^{\prime} \sim \mathfrak{M}$ and $\mathscr{D} \mid \tau^{\prime \prime} \sim \mathfrak{R}$. Logic $L$ has the Robinson property (or, $L$ is a Robinson logic) iff $L$ satisfies Robinson's consistency theorem, i.e., iff $\equiv_{L}$ is a Robinson equivalence relation. (Compare with [Mu1].) Notice that $\tau$, $\tau^{\prime}$ and $\tau^{\prime \prime}$ need not all have the same set of sorts.

The importance of Robinson logics and equivalence relations is evident from [Mu1-Mu6] (but see also [MS, postscriptum] and [MS1]); further nice properties are given by the results in this paper.

\section{The union of Robinson logics.}

2.1 Proposition. Let $\left\{L_{i}\right\}_{i \in I}$ be a class of logics; let $L=\cup_{i \in I} L_{i}$ be their union, i.e. the weakest logic which is stronger than each $L_{i}$; then for any $\tau$ and $\varphi \in \operatorname{Stc}_{L}(\tau), \varphi$ has the same models as $\psi$, where

$$
\psi=Q_{1} x_{1} \cdots Q_{n} x_{n} B\left(\varphi_{1}^{1}, \ldots, \varphi_{p_{1}}^{1} ; \ldots ; \varphi_{1}^{t}, \ldots, \varphi_{p_{t}}^{t}\right)
$$

where each $Q_{z}$ is either $\exists$ or $\forall$ (depending on $z$ ), B is a boolean combination of a finite number of sentences (depending on $\varphi$ ) of type $\tau \cup\left\{x_{1}, \ldots, x_{n}\right\}$ and any two $\varphi$ 's having the same upper index belong to the same $L_{i}$. Conversely, each $\psi$ as given by $(+)$ is equivalent to a sentence in $L$.

Proof. Clearly, for any $\psi$ given by $(+)$ above there must be in $L$ some sentence equivalent to $\psi$, as $L$ is closed under the boolean operations and quantification by $\exists$ and $\forall$. Thus we have only to show that the class of sentences given by $(+)$ is closed under the boolean operations, existential quantification and relativization; as a matter of fact, closure under $\exists$ is trivial; closure under boolean operations and relativization may be proved in a tedious but straightforward way by induction on the complexity of the prefix in $(+)$, upon recalling the usual (high school) gymnastics about pushing quantifiers to the left. Q.E.D. 
2.2 Proposition. Let $L_{1}, \ldots, L_{k}$ be Robinson logics with $\equiv_{L_{1}}=\ldots=\equiv_{L_{k}}$. Let $\varphi$ be given by

$$
\varphi=Q_{1} x_{1} \ldots Q_{n} x_{n} B\left(\varphi_{1}^{1}(\vec{x}), \ldots, \varphi_{p_{1}}^{1}(\vec{x}) ; \ldots ; \varphi_{1}^{k}(\vec{x}), \ldots, \varphi_{p_{k}}^{k}(\vec{x})\right)
$$

where $\vec{x}=\left(x_{1}, \ldots, x_{n}\right)$, the $Q_{z}$ and $B$ are as in the statement of 2.1 , and, for $1 \leqslant r \leqslant p_{j}, \varphi_{r}^{j} \in \operatorname{Stc}_{L_{j}}\left(\tau \cup\left\{x_{1}, \ldots, x_{n}\right\}\right)$. Then for any $\mathfrak{A}, \mathfrak{B} \in \operatorname{Str}(\tau)$ with $\mathfrak{A} \equiv_{L_{1}} \mathfrak{B}$ we have that $\mathfrak{A}$ satisfies $\varphi$ iff $\mathfrak{B}$ satisfies $\varphi\left(\right.$ in $\left.L=L_{1} \cup \ldots \cup L_{k}\right)$.

Proof. Deny (absurdum hypothesis); expand $\mathfrak{A}$ to $\mathfrak{A}^{+}$by adding new $n$-ary relation symbols $U_{1}^{1}, \ldots, U_{p_{1}}^{1} ; \ldots ; U_{1}^{k}, \ldots, U_{p_{k}}^{k}$ which are interpreted in model $\mathfrak{A}^{+}$by

$$
\mathfrak{A}^{+} \vDash_{L} \forall \vec{x}\left(U_{i}^{j}(\vec{x}) \leftrightarrow \varphi_{i}^{j}(\vec{x})\right)
$$

for any $j=1, \ldots, k$ and $i=1, \ldots, p_{j}$. Then from $\mathfrak{A}_{L} \varphi$ we get

$$
\mathfrak{A}^{+} F_{L} Q_{1} x_{1} \ldots Q_{n} x_{n} B\left(U_{1}^{1}(\vec{x}), \ldots, U_{p_{1}}^{1}(\vec{x}) ; \ldots ; U_{1}^{k}(\vec{x}), \ldots, U_{p_{k}}^{k}(\vec{x})\right) .
$$

Now let $\mathfrak{M} \equiv \equiv_{L_{1}} \mathfrak{A}^{+}$; then

$$
\mathfrak{M} \equiv{ }_{L_{j}} \mathfrak{A}^{+} \quad \text { for any } j=1, \ldots, k
$$

by hypothesis; hence we get from (1) and (3):

$$
\mathfrak{M F}_{L} \forall \vec{x}\left(U_{i}^{j}(\vec{x}) \leftrightarrow \varphi_{i}^{j}(\vec{x})\right)
$$

for any $j=1, \ldots, k$ and $i=1, \ldots, p_{j}$; by (2) and definition of $\mathfrak{M}$ we get

$$
\mathfrak{M k}_{L} Q_{1} x_{1} \ldots Q_{n} x_{n} B\left(U_{1}^{1}(\vec{x}), \ldots, U_{p_{1}}^{1}(\vec{x}) ; \ldots ; U_{1}^{k}(\vec{x}), \ldots, U_{p_{k}}^{k}(\vec{x})\right) ;
$$

and from (5) and (4) we finally have

$$
\mathfrak{M} \vDash_{L} \varphi .
$$

This shows that $\bmod _{L_{1}} \operatorname{th}_{L_{1}} \mathfrak{U}^{+} \subseteq \bmod _{L} \varphi$ (by 2.1 we can safely assume that $\varphi \in \operatorname{Stc}_{L}(\tau)$ ). By a similar argument, if we expand $\mathfrak{B}$, where $\mathfrak{B} \vDash_{L} \neg \varphi$, to $\mathfrak{B}^{+}$by adding new $n$-ary relation symbols $V_{1}^{1}, \ldots, V_{p_{k}}^{k}$ then we get $\bmod _{L_{1}} \operatorname{th}_{L_{1}} \mathfrak{B}^{+} \subseteq$ $\bmod _{L} \neg \varphi$. Now by noting that the common symbols of $\mathfrak{A}^{+}$and $\mathfrak{B}^{+}$are in $\tau$, and by applying Robinson's consistency theorem to $L_{1}$, one concludes that $\mathfrak{A}$ and $\mathfrak{B}$ are not $L_{1}$-elementarily equivalent, a contradiction. Q.E.D.

2.3 Proposition. Let $\left\{L_{i}\right\}_{i \in I}$ be a class of Robinson logics such that $\equiv_{L_{i}}=\equiv_{L_{j}}$ for all $i, j \in I$; let $L$ be their union; then $\equiv_{L}=\equiv_{L_{i}}$ for all $i \in I$ and, in particular, $L$ is still a Robinson logic.

Proof. Each sentence in $L$ is given (up to equivalence) by $(+)$ in 2.1 ; now no such sentence can "separate" two structures $\mathfrak{U}$ and $\mathfrak{B}$ with $\mathfrak{A} \equiv_{L_{i}} \mathfrak{B}$, by Proposition 2.2; this shows that $\equiv_{L}=\equiv_{L_{i}}$; the fact that $L$ is still a Robinson logic now immediately follows by noting that this only depends on $\equiv_{L}$. Q.E.D.

2.4 REMARK. In the following sections we shall study the uniqueness and the existence problems for representations of equivalence relation $\sim$ as $\equiv_{L}$, with $L$ a logic. We shall consider both the infinitary case (\$3) and the case when $\left|\operatorname{Stc}_{L} \tau\right|$ exists for any $\tau(\$ \S 4-6)$; for this latter case we shall obtain a complete solution of the representation problem in Theorem 5.5 below. 


\section{A general theorem.}

3.1 TheOREM. (Assuming $\omega$ is the only measurable cardinal): Let $\sim$ be a Robinson equivalence relation which is representable as $\equiv_{L}$. for $L \leqslant L^{*}$ a logic (with $L^{*}$ arbitrary); then there exists a strongest logic $L^{+} \leqslant L^{*}$ with $\equiv_{L^{+}}=\sim$; furthermore, $L^{+}$is countably compact (indeed, $\omega-$ r.c.) if $\cong \neq \sim$.

Proof. The existence of a strongest logic $L^{+}$follows from the representability of $\sim$ and from Proposition 2.3, letting $L^{+}=$union of the logics $L^{\prime \prime} \leqslant L^{*}$ with $\equiv_{L^{\prime \prime}}=\sim$; for the proof of the second part of the theorem we prepare:

3.2 Lemma. Let $\nu$ be an arbitrary but fixed infinite cardinal; let $L$ be a Robinson logic which is not $\kappa$-r.c. for all $\kappa<\nu$; then every single-sorted structure $\mathfrak{A}$ with $|A|<\nu$ is characterizable by its theory in $L$.

Proof of 3.2. See [Mu5, Corollary 4.2]; alternatively, extend up to $\nu$ the constructions given by $[\mathbf{M u 2}, 2.1,1.6,1.2]$, which can be done in a straightforward manner. Notice that only relativization to atomic formulas is used in [Mu2] and [Mu5].

3.3 Lemma. (Assuming $\omega$ is the only measurable cardinal): if $L$ is a Robinson logic which is not countably compact, or which is not w-r.c., then every single-sorted structure is characterizable by its theory in $L$.

Proof of 3.3. In view of Lemma 3.2 above, it suffices to prove that $L$ is not $\kappa$-r.c. for each cardinal $\kappa \geqslant \omega$. If not (absurdum hypothesis) let $\mu$ be the least cardinal such that $L$ is $\mu$-r.c.; notice that $\mu>\omega$ since otherwise $L$ would also be countably compact (letting $\Gamma=\varnothing$ in the definition of $\kappa$-r.c.) thus contradicting our assumptions. By Lemma 3.2 each ordinal $\beta<\mu$ is characterizable in its theory in type $\{<\}$ with $<$ a binary relation symbol. Define a single-sorted type $\tau$ by

$$
\tau=\left\{<, c_{\beta}, P_{s}, f_{g}\right\} \quad \text { for all } \beta<\mu, s \in \mathscr{P}(\mu), g \in \in^{\mu} \mu,
$$

where $P$ denotes power set and the $c$ 's, the $P$ 's and the $f$ 's are constant, unary relation and unary function symbols, respectively. Let $\mathfrak{M} \in \operatorname{Str}(\tau)$ be defined by

$$
\begin{aligned}
& \mathfrak{M} \vDash\left\{<, c_{\beta}\right\}_{\beta<\mu}=\langle\mu,<, \beta\rangle_{\beta<\mu}, \\
& \mathfrak{M} \vDash P_{s}\left(c_{\beta}\right) \quad \text { iff } \beta \in s, \text { for each } \beta<\mu, s \in \mathscr{P}(\mu), \\
& \mathfrak{M} \vDash f_{g}\left(c_{\alpha}\right)=c_{\beta} \text { iff } g(\alpha)=\beta, \text { for each } \alpha, \beta<\mu, g: \mu \rightarrow \mu .
\end{aligned}
$$

Let $\Gamma=\mathrm{th}_{L} \mathfrak{M}$ and $\Sigma=\left\{c>c_{\alpha} \mid \alpha<\mu\right\}$ with $c$ a new constant symbol; since $L$ is $\mu$-r.c. then $\Gamma \cup \Sigma$ is consistent; so let $\mathfrak{M}^{+} \vDash \Gamma \cup \Sigma$ (we drop subscript $L$ since no confusion may arise). Without loss of generality we also assume that

$$
\mathfrak{M}<_{L_{\omega \omega}} \mathfrak{M I}^{+},
$$

i.e. $\mathfrak{M}$ is an elementary substructure of $\mathfrak{M}^{+}$. Compare with [MS, 6.4(ii)] and [MS1, 2.6].

Let $D \subseteq \stackrel{P}{ }(\mu)$ be given by

$$
d \in D \quad \text { iff } \mathfrak{M}^{+} \vDash P_{d}(c), \quad \text { for } d \in \mathscr{P}(\mu) .
$$


Roughly, the elements of $D$ are the restrictions on $\mu$ of the subsets of the universe of $\mathfrak{M}^{+}$having a "name" in $\mathfrak{M}$ and to which $c$ belongs (in $\mathfrak{M}^{+}$). One sees that $D$ is a nonprincipal ultrafilter on $\mu$, by using (1) and (2); we propose to show that $D$ is $\mu$-complete, thus contradicting our set-theoretical assumption. If not, $D$ is $\lambda$ descendingly incomplete for some $\lambda<\mu$ (see [CK, Exercise 4.3.10(iii)]), i.e. there exists a decreasing chain $D^{\prime}=\left\{d_{\alpha}\right\}_{\alpha<\lambda}$ of elements of $D$ such that $\bigcap_{\alpha<\lambda} d_{\alpha} \notin D$, i.e., without loss of generality, $\bigcap_{\alpha<\lambda} d_{\alpha}=\varnothing$. Therefore, we can define $h: \mu \rightarrow \lambda$ by stipulating that

$$
h(\beta)=\alpha \quad \text { iff } \beta \in d_{\alpha} \backslash d_{\alpha+1} \quad(\beta<\mu, \alpha<\lambda) ;
$$

roughly, $h(\beta)$ says how long $\beta$ remains in (the elements of) $D^{\prime}$. Let now $f=f_{h}$, $P_{\alpha}=P_{d_{\alpha}}(\alpha<\lambda)$; these are symbols in $\tau$. Then we have:

$$
\begin{aligned}
& \forall \alpha<\lambda, \mathfrak{M} \vDash \forall x\left(f(x) \leqslant c_{\alpha} \rightarrow \neg P_{\alpha+1}(x)\right) ; \\
& \forall \alpha<\lambda, \mathfrak{M}^{+} \vDash \forall x\left(f(x) \leqslant c_{\alpha} \rightarrow \neg P_{\alpha+1}(x)\right) \quad(\text { by }(1)) ; \\
& \forall \alpha<\lambda, \mathfrak{M}^{+} \vDash f(c) \leqslant c_{\alpha} \rightarrow \neg P_{\alpha+1}(c) ; \\
& \forall \alpha<\lambda, \mathfrak{M}^{+} \vDash f(c)>c_{\alpha}, \text { as } \mathfrak{M}^{+} \vDash P_{\alpha+1}(c) \quad \text { by }(2) ; \\
& \mathfrak{M}^{+} \vDash \forall x\left(x<c_{\lambda} \rightarrow f(c)>x\right) \quad(\text { since } \lambda \text { is characterizable); } \\
& \mathfrak{M}^{+} \vDash \exists y \forall x\left(x<c_{\lambda} \rightarrow f(y)>x\right) ; \\
& \mathfrak{M}^{*} \vDash y \forall x\left(x<c_{\lambda} \rightarrow f(y)>x\right) \quad(\text { by }(1)) ; \\
& \bigcap d_{\alpha} \neq \varnothing .
\end{aligned}
$$

Thus $D$ is indeed $\mu$-complete and $\mu$ is measurable $>\omega$, a contradiction with our set-theoretical hypothesis. In definitive, we conclude that $L$ is never $\kappa$-r.c. Q.E.D.

3.4 LEMMA. Let $L$ be a Robinson logic in which each single-sorted structure is characterizable up to isomorphism by its theory; then each (many-sorted) structure is characterizable up to isomorphism by its theory in $L$.

Proof of Lemma 3.4. Deny (absurdum hypothesis); so let $\mathfrak{M}$ and $\mathfrak{R}$ be two many-sorted structures of type $\tau$ with $\mathfrak{M} \equiv_{L} \mathfrak{N}$ but $\mathfrak{M} \not \mathfrak{N}$. Add a new unary relation symbol $U_{s}$ for each sort $s \in \tau$; recall that $\tau$ is a set; represent $\mathfrak{M}$ as a one-sorted structure $\mathfrak{M}^{\prime}$ by letting

$$
\begin{gathered}
\text { universe of } \mathfrak{M}^{\prime}=\text { union of the universes of the sorts of } \mathfrak{M}, \\
U_{s}^{\mathfrak{M}^{\prime}}=\text { universe of sort } s \text { in } \mathfrak{M} \quad \text { (for any sort } s \in \tau \text { ). }
\end{gathered}
$$

For simplicity assume $\tau$ has no function symbol; let $i_{s}$ be the identity map from the universe of sort $s$ in $\mathfrak{M}$ onto $U_{s}^{\mathfrak{M}^{\prime}}$; let $i=\cup_{s \in \tau} i_{s}$; let the symbols of $\tau$ be interpreted in $\mathfrak{M}^{\prime}$ according to the canonical relativization induced by $i$ (see [Mo, $\mathrm{p}$. 484] for a similar construction); also assume that each $R \in \tau$ is written $R^{\prime}$ in $\mathfrak{M}^{\prime}$ : let $\tau^{\prime}$ be the set of primed symbols thus obtained. Roughly, $i$ induces a one-sorted photograph $\mathfrak{M}^{\prime}$ of the many-sorted structure $\mathfrak{M}$. Similarly, let $\mathfrak{N}^{\prime \prime}$ be a one-sorted representation of $\mathfrak{N}$ induced by function $j$, where new unary relation symbols $V_{s}$ are used to relativize sort $s$ and each $R \in \tau$ is written as $R^{\prime \prime}$ in $\mathfrak{R}^{\prime \prime}$ : let $\tau^{\prime \prime}$ be the set of 
doubly primed symbols thus obtained. Let $\rho$ be a name-changer which maps each $R^{\prime} \in \tau^{\prime}$ into $R^{\prime \prime} \in \tau^{\prime \prime}$ and maps each $U_{s}$ into $V_{s}$; let $\left(\mathfrak{M}^{\prime}\right)^{\rho}$ be the structure canonically obtained from $\mathfrak{M}^{\prime}$ via $\rho$; notice that

$$
\left(\mathfrak{M}^{\prime}\right)^{\rho} \not \mathfrak{N}^{\prime \prime}
$$

as $\mathfrak{M} \neq \mathfrak{N}$; thus, by hypothesis about $L$ applied to the single-sorted structures $\mathfrak{M}^{\prime}$ and $\mathfrak{R}^{\prime \prime}$, we get

$$
\left(\mathfrak{M}^{\prime}\right)^{\rho} Z_{L} \mathfrak{N}^{\prime \prime}
$$

Let now $\mathfrak{U}=\left\langle\mathfrak{M}, \mathfrak{M}^{\prime}, i\right\rangle$ and $\mathfrak{B}=\left\langle\mathfrak{R}, \mathfrak{R}^{\prime \prime}, j\right\rangle$. Recalling that $\mathfrak{M} \equiv_{L} \mathfrak{R}$, by the assumed Robinson consistency theorem for $L$ there exists $\mathscr{D}$ which is both $L$ equivalent to $\mathfrak{A}$ and to $\mathfrak{B}$, hence, in particular (by the basic expansion axiom of logics),

$$
\mathscr{D} / \tau^{\prime} \equiv_{L} \mathfrak{M}^{\prime} \text { and } \mathscr{D} / \tau^{\prime \prime} \equiv_{L} \Re^{\prime \prime} \text {. }
$$

Therefore, by the renaming axiom of logics and by (2) we can write

$$
\left(\mathscr{D} \mid \tau^{\prime}\right)^{\rho} z_{L} \mathfrak{D} \mid \tau^{\prime \prime}
$$

hence, by the isomorphism axiom logics, we have

$$
\left(\mathfrak{D} \mid \tau^{\prime}\right)^{\rho} \not \mathfrak{D} \mid \tau^{\prime \prime} .
$$

Now look at $\mathscr{D} \mid \tau: i$ and $j$ induce two one-sorted representations of $\mathscr{D} \mid \tau$ which we write as $i(\mathfrak{D} \mid \tau)$ and $j(\mathfrak{D} \mid \tau)$, respectively, of type $\tau^{\prime}$ and $\tau^{\prime \prime}$; since $i$ and $j$ are structure-preserving, then we have

$$
(i(\mathfrak{D} \mid \tau))^{\rho} \cong j(\mathfrak{D} \mid \tau) .
$$

In view of the fact that $i(\mathscr{D} \mid \tau)=\mathscr{D} \mid \tau^{\prime}$ and $j(\mathfrak{D} \mid \tau)=\mathscr{D} \mid \tau^{\prime \prime}$, we finally see that (5) and (6) contradict each other. Q.E.D.

3.5 END OF THE PROOF. Let $L^{+}$be the strongest logic such that $\equiv_{L^{+}}=\sim$; if $L^{+}$is not countably compact, then by 3.3 and 3.4 it follows that each structure is characterizable in $L^{+}$by its theory, i.e. $\equiv_{L^{+}}=\cong$. Q.E.D. ${ }^{1}$

3.6 Corollary. (Assuming $\omega$ is the only measurable cardinal): Let $\sim$ be a representable Robinson equivalence relation; then the following are equivalent:

(i) $\sim$ characterizes $\langle\omega,<\rangle$, i.e. $I\langle\omega,<\rangle=\{\mathfrak{R} \mid \mathfrak{R} \sim\langle\omega,<\rangle\}$;

(ii) $\sim$ characterizes every structure, viz. $\sim=\cong$.

Proof. (ii) $\rightarrow$ (i) is trivial; to prove the converse, letting $L$ be such that $\sim=\equiv_{L}$, we immediately see that $L$ cannot be $\omega$-r.c. (by a familiar argument which can be found, among others, in [MS]); therefore, by 3.3 and 3.4 we have that $\cong=\sim$. Q.E.D.

3.7 Remark. Compare with [Mu4, Theorem 4], which essentially proves the above corollary upon restriction to countable structures only.

\footnotetext{
${ }^{1}$ Matt Kaufmann has given a simple proof of a many-sorted version of 3.2, thus eliminating 3.4 from the proof of 3.1. He also posed the problem whether one can find a counterexample to 3.1 if it is no longer assumed that $\sim$ is Robinson. He finally remarked that from [MS1, 5.8, 5.9] one can see that some large cardinal hypothesis is actually necessary for Theorem 3.1 above to hold.
} 
4. A duality theorem. Theorem 3.1 can be further improved if we assume that has not too many equivalence classes of structures of type $\tau$; more precisely, we stipulate:

4.1 Definition. We say that an equivalence relation $\sim$ on the class of all structures is bounded iff for any type $\tau$ the collection of the equivalence classes of structures of type $\tau$ is equinumerous with some cardinal $\kappa_{\tau}$.

In other words, $\sim$ is bounded iff for every type $\tau$ there is a set $S_{\tau} \subseteq \operatorname{Str}(\tau)$ such that $\forall \mathfrak{A} \in \operatorname{Str}(\tau) \exists \mathfrak{B} \in S_{\tau}$ with $\mathfrak{B} \sim \mathfrak{A}$. Compare with [Na]; notice here that our notion of a logic incorporates from the start closure under first-order operations, including relativization.

For bounded equivalence relations we have the following:

4.2 Theorem (Assuming $\omega$ is the only measurable cardinal): Let $\sim$ be a bounded Robinson equivalence relation; assume $\equiv_{L^{\prime}}=\sim=\equiv_{L^{\prime \prime}}$; then $L^{\prime}=L^{\prime \prime}$ and both are compact logics obeying Craig's interpolation.

Proof. First notice that since $\sim$ is bounded, then for any type $\tau$ the collection of elementary classes of type $\tau$ in $L^{\prime}$ is equinumerous with a set $G_{\tau}$; also, $L^{\prime}$ satisfies Robinson's consistency theorem as so does $\equiv_{L^{\prime}}$. Therefore we can apply the corollary to the main theorem in [Mu3], to the effect that $L^{\prime}$ is compact and obeys Craig's interpolation; the same applies to $L^{\prime \prime}$; let $L=L^{\prime} \cup L^{\prime \prime}$; for each $\tau$, in $L$ the elementary classes of type $\tau$ are equinumerous with a set $H_{\tau}$, by Proposition 2.1, and $\equiv_{L}=\equiv_{L^{\prime}}$, by Proposition 2.3, so that $L$ satisfies Robinson's consistency theorem as well; again by applying the corollary to the main theorem in [Mu3], we see that $L$ is compact; now, since $L \geqslant L^{\prime}$, by a familiar (finite cover) argument found, e.g., in [Fl], we have that $L=L^{\prime}$ (notice that here one needs the fact that the collection of elementary classes in $L^{\prime}$ of type $\tau$ is equinumerous with a set, in order to be able to exploit compactness); similarly, $L=L^{\prime \prime}$. Notice that in [Mu3] only relativization to atomic formulas is actually used. Q.E.D.

4.3 REMARKs. The boundedness assumption is clearly necessary: consider, for instance, $L_{\infty \infty}$ and any logic stronger than it. The assumption that $\omega$ is the only measurable cardinal is used for the proof of the results in [Mu3] on which the proof of 4.2 depends.

By the above theorem we see that the function $L \mapsto \equiv_{L}$ maps the class of all logics satisfying Robinson's consistency and having a bounded number of sentences (mod equivalence) in every type, one-one onto the class of all the representable bounded Robinson equivalence relations (assuming $\omega$ is the only measurable cardinal). In the next section we shall give a necessary and sufficient condition for an equivalence relation to be representable by some logic $L$ satisfying compactness and Craig's interpolation.

In this sense, one will be then able to speak of a "duality" between logics and equivalence relations.

4.4 Corollary. (Assuming $\omega$ is the only measurable cardinal): There exists just one logic L such that $\equiv_{L}=\equiv$, namely first-order logic. 
Proof. $\equiv$ is representable (e.g., via first-order logic $L_{\omega \omega}$ ); further, $\equiv$ is a Robinson equivalence relation, by the familiar Robinson's consistency theorem in first-order logic (see [CK]); finally, $\equiv$ is bounded, as can be immediately seen by considering that $\left|\operatorname{Stc}_{L_{\omega \omega}}(\tau)\right|=|\tau \cup \omega|$. Now apply Theorem 4.2. Q.E.D.

4.5 RemarK. One can see without difficulty that the (generalized downward) Löwenheim-Skolem theorem together with Lindström's theorem (see [Li] or [FI]) directly yield that $L_{\omega \omega}$ is the only compact logic $L$ such that $\equiv_{L}=\equiv$ (one might even use a simpler "finite cover" argument). Also notice that the collection of all unions of classes of elementarily equivalent structures of type $\tau$, with $\tau$ arbitrary, does not give (the elementary classes of) a logic, since it is not closed under existential quantification; if one attempts to close this collection under all the first-order operations, the resulting logic $L^{\prime}$ has $\equiv_{L^{\prime}} \neq \equiv$.

5. Characterizing representability. In this section we shall state the characterization theorem for those equivalence relations $\sim$ which are equal to $\equiv_{L}$ for some compact $\operatorname{logic} L$ obeying Craig's interpolation. The proof will be given in the next section. For our characterization we shall need to construct logic $L$ representing $\sim$ : to get such $L$ one first studies a system of classes of structures which is closed under all the first-order operations and which is, in some sense, generated by $\sim$; the necessary set up is given by the following discussion.

A finite-type class $X$ is an ordered pair $X=(S, \tau)$ where $\tau$ is a finite type and $S \subseteq \operatorname{Str}(\tau)$; we naturally say that $X$ is elementary iff $S=\bmod \varphi$ for some first-order sentence $\varphi$ of type $\tau$; we incorporate $\tau$ in the definition of $X$ so that we can unambiguously speak of the type of $X$, for short $\tau(X)$ : actually this becomes critical only for classes of the form $X=(\varnothing, \tau)$. In any case we shall freely write $\mathfrak{A} \in X$ to mean $\mathfrak{U} \in S$.

For $\rho: \tau \rightarrow \rho(\tau)$ a name-changer, $\rho$ acts on $X$ yielding $\rho X$ of type $\rho(\tau)$ defined by

$$
\mathfrak{A} \in \rho X \quad \text { iff } \mathfrak{H}^{\rho}{ }^{1} \in X .
$$

Given $X=(S, \tau)$, the negation of $X$ is $\neg X=(\operatorname{Str}(\tau) \backslash S, \tau)$; for $X_{1}=\left(S_{1}, \tau_{1}\right)$ and $X_{2}=\left(S_{2}, \tau_{2}\right)$, the conjunction $X_{1} \wedge X_{2}$ is given by

$\mathfrak{A} \in X_{1} \wedge X_{2} \quad$ iff $\mathfrak{A} \in \operatorname{Str}\left(\tau_{1} \cup \tau_{2}\right)$ and $\mathfrak{A} \mid \tau_{1} \in X_{1}$ and $\mathfrak{A} \uparrow \tau_{2} \in X_{2}$.

The disjunction $X_{1} \vee X_{2}$ is defined as $\neg\left(\neg X_{1} \wedge \neg X_{2}\right)$, and is a finite-type class of type $\tau_{1} \cup \tau_{2}$.

For $b$ a constant symbol and $X=(S, \tau)$, the projector $\exists b$ transforms $X$ into $\exists b X$ of type $\tau \backslash\{b\}$ given by

$$
\mathscr{A} \in \exists b X \text { iff } \mathfrak{A} \in \operatorname{Str} \tau \backslash\{b\} \text { and } \mathscr{H} \text { has an expansion } \mathfrak{A}^{+} \in X .
$$

We also let $\forall b X$ be defined as $\neg \exists b \neg X$. A prenex function $Q_{1} k_{1} \ldots Q_{n} k_{n}$, for short $\vec{Q} \circ \vec{k}$, where each $Q_{i}$ is either $\exists$ or $\forall$, depending on $i$, and each $k_{i}$ is a constant symbol, transforms $X$ into $\vec{Q} \circ \vec{k} X$ given by

$$
\vec{Q} \circ \vec{k} X=Q_{1} k_{1}\left(\ldots\left(Q_{n} k_{n} X\right) \ldots\right) .
$$

We now consider relativization: in the single-sorted case, see $[\mathbf{F I}]$, the notion of $\mathfrak{A}$ being $\tau$-closed upon restriction to $\{x \mid U(x)\}$ means that in $\mathfrak{A}$ : (i) $U(x)$ has some 
solution, (ii) each constant of $\tau$ satisfies $U(x)$, and (iii) the output of each function symbol $f \in \tau$ satisfies $U(x)$ whenever (each component of) its input does. Also, the models of the relativization $\varphi^{U}$ of sentence $\varphi$ of type $\tau$ to $\{x \mid U(x)\}$ are the structures of type $\tau \cup\{U\}$ which are $\tau$-closed upon restriction to $\{x \mid U(x)\}$ and such that $\mathfrak{A} \mid\{x \mid U(x)\}$ is a model of $\varphi$. Notice that we incorporate $\tau$-closure in the definition of relativization.

The above is naturally generalized to the many-sorted case as follows: let $\tau$ be a finite type with sorts $s_{1}, \ldots, s_{z}$; let $\vec{x}=x^{1}, \ldots, x^{2}$ be a sequence of constants, respectively, of sorts $s_{1}, \ldots, s_{z}$; let $\vec{\psi}=\psi_{1}, \ldots, \psi_{z}$ be a sequence where each $\psi_{i}$ is a boolean combination of atomic sentences and is of type $\tau_{i}$ containing $x^{i}$. Then the class

$$
C_{\tau}^{\{\vec{x} \mid \vec{\psi}\}} \subseteq \operatorname{Str} \tau \cup \tau_{1} \backslash\left\{x^{1}\right\} \cup \ldots \cup \tau_{z} \backslash\left\{x^{z}\right\}
$$

of the structures which are $\tau$-closed upon restriction to $\{\vec{x} \mid \vec{\psi}\}$ is defined by

$\mathfrak{A} \in C_{\tau}^{\{\vec{x} \mid \vec{\psi}\}}$ iff the (many-sorted) universe $A^{\prime} \subseteq A$ given by

$$
A_{1}^{\prime}=\left\{a^{1} \in A \mid \mathfrak{A} \vDash \psi_{1}\left(a^{1}\right)\right\}, \ldots, A_{z}^{\prime}=\left\{a^{z} \in A \mid \mathfrak{A} \vDash \psi_{z}\left(a^{z}\right)\right\}
$$

has the following properties in $\mathfrak{A}$ :

(i) is nonempty on each sort of $\tau$, i.e., $A_{1}^{\prime}, \ldots, A_{z}^{\prime} \neq \varnothing$;

(ii) contains (the interpretations of) the constants of $\tau$;

(iii) contains the output $f(k)$ of each $f \in \tau$ whenever the input $k$ (has the right sorts for $f$ and) has its components in $A^{\prime}$.

Obviously, since $\tau$ is finite, $C_{\tau}^{\{\vec{x} \psi\}}$ is an elementary class. Under the same notation, for $X$ a finite-type class of type $\tau$,

$$
X^{\{\vec{x} \mid \vec{\psi}\}} \subseteq \operatorname{Str} \tau \cup \tau_{1} \backslash\left\{x^{1}\right\} \cup \ldots \cup \tau_{z} \backslash\left\{x^{z}\right\}
$$

called the relativization of $X$ to $\{\vec{x} \mid \vec{\psi}\}$, is the finite-type class given by

$$
\mathfrak{U} \in X^{\{\vec{x} \mid \vec{\psi}\}} \quad \text { iff } \mathfrak{U} \in C_{\tau}^{\{\vec{x} \mid \vec{\psi}\}} \text { and } \mathfrak{U} \uparrow \tau \mid A^{\prime} \in X,
$$

where $\mathfrak{A} \uparrow \tau \mid A^{\prime}$ is the substructure of $\mathfrak{A} \uparrow \tau$ generated by $A^{\prime}$.

Again notice that $\tau$-closure is incorporated in relativization. The main properties of relativization used below are summarized in the following

5.1 Proposition. Let $X, \tau, \vec{x}=x^{1}, \ldots, x^{z} ; \vec{\psi}=\psi_{1}, \ldots, \psi_{z}, s_{1}, \ldots, s_{z}, \tau_{1}, \ldots, \tau_{z}$ be as above; then

(i)

$$
(\neg X)^{\{x \mid \vec{\psi}\}}=C_{\tau}^{\{\vec{x} \vec{\psi}\}} \wedge \neg\left(X^{\{\vec{x} \mid \vec{\psi}\}}\right) ;
$$

(ii) for $\rho$ any name-changer, $\rho\left(X^{\{\vec{x} \mid \vec{\psi}\}}\right)=\left(\rho^{\prime} X\right)^{\{\vec{v} \mid \vec{\varphi}\}}$, where $\rho^{\prime}$ is the restriction of $\rho$ to $\tau, \vec{y}=y^{1} \ldots y^{z}$ are new constants of sort $\rho\left(s_{1}\right), \ldots, \rho\left(s_{z}\right)$, respectively, $\vec{\varphi}=\varphi_{1}, \ldots, \varphi_{z}$ where $\varphi_{i}$ is obtained from $\psi_{i}$ by writing $y^{i}$ instead of $x^{i}$, and by mapping any other symbol $S$ of $\tau_{i}$ into $\rho(S)$;

(iii) for $h$ a constant of sort $s_{i}(1 \leqslant i \leqslant z)$, we have

$$
(\exists h X)^{\{\vec{x} \mid \vec{\psi}\}}=\exists h^{\prime}\left(\psi_{i}^{\prime} \wedge X^{\prime\{\vec{x} \mid \vec{\psi}\}}\right)
$$


and

$$
(\forall h X)^{\{\vec{x} \mid \vec{\psi}\}}=(\neg \exists h \neg X)^{\{\vec{x} \mid \vec{\psi}\}}=C_{\tau^{\prime}}^{\{\vec{x} \mid \vec{\psi}\}} \wedge \forall h^{\prime}\left(\psi_{i}^{\prime} \rightarrow X^{\prime\{\vec{x} \mid \vec{\psi}\}}\right)
$$

where $\psi_{i}^{\prime}$ and $X^{\prime}$ are obtained from $\psi_{i}$ and $X$ upon renamings $x^{i} \mapsto h^{\prime}$ and $h \mapsto h^{\prime}$, respectively, for $h^{\prime}$ a suitable new constant of sort $s_{i}$, and $\tau^{\prime}=\tau \backslash\{h\}$;

(iv) for classes $X^{\prime}$ and $X^{\prime \prime}$, respectively, of type $\tau^{\prime}$ and $\tau^{\prime \prime}$, with $\tau^{\prime} \cup \tau^{\prime \prime}=\tau$, we have

$$
\begin{gathered}
\left(X^{\prime} \wedge X^{\prime \prime}\right)^{\{\vec{x} \mid \vec{\psi}\}}=C_{\tau}^{\{\vec{x} \mid \vec{\psi}\}} \wedge X^{\prime\{\vec{x} \mid \vec{\psi}\}^{\prime}} \wedge X^{\prime \prime\left\{(\vec{x} \vec{\psi}\}^{\prime \prime}\right.}, \\
\left(X^{\prime} \vee X^{\prime \prime}\right)^{\{\vec{x} \mid \vec{\psi}\}}=\left(\neg\left(\neg X^{\prime} \wedge \neg X^{\prime \prime}\right)\right)^{\{\vec{x} \mid \vec{\psi}\}}=C_{\tau}^{\{\vec{x} \mid \vec{\psi}\}} \wedge\left(X^{\prime\{\vec{x} \vec{\psi}\}^{\prime}} \vee X^{\prime \prime\{\vec{x} \mid \vec{\psi}\}^{\prime \prime}}\right),
\end{gathered}
$$

where $\{\vec{x} \mid \vec{\psi}\}^{\prime}\left(\right.$ resp. $\left.\{\vec{x} \mid \vec{\psi}\}^{\prime \prime}\right)$ is obtained from $\{\vec{x} \mid \vec{\psi}\}$ by leaving only those $i$ such that $s_{i} \in \tau^{\prime}\left(\right.$ resp. $\left.s_{i} \in \tau^{\prime \prime}\right)$.

(v) if $\vec{y}=y^{1}, \ldots, y^{w}$ are constants of sort $s_{1}, \ldots, s_{w}$, respectively $(w \geqslant z)$, and $\vec{\varphi}=\varphi_{1}, \ldots, \varphi_{w}$ is a sequence where each $\varphi_{i}$ is a boolean combination of atomic sentences, of type $\tau_{\dot{\phi}}$ containing $y^{i}$, and the sorts of $\tau \cup \tau_{1} \cup \ldots \cup \tau_{z}$ are $s_{1}, \ldots, s_{w}$, then $\left(X^{\{\vec{x} \vec{\psi}\}}\right)^{\{\overrightarrow{\mid \vec{\varphi}}\}}=X^{\{t \vec{x}\}} \wedge F$, where $\vec{t}=t^{1}, \ldots, t^{z}$ is a sequence of constants of sort $s_{1}, \ldots, s_{z}$, respectively, $F$ is elementary, expressing some closure property, $\chi_{j}$ is $\varphi_{j}^{\prime} \wedge \psi_{j}^{\prime}$, where $\psi_{j}^{\prime}$ is obtained from $\psi_{j}$ by renaming $x^{j} \mapsto t^{j}$ and $\varphi_{j}^{\prime}$ is obtained from $\varphi_{j}$ by renaming $y^{j} \mapsto t^{j}, j=1, \ldots, z$.

Proof. By a tedious but direct application of the definitions. Q.E.D.

Having defined the first-order operations on classes of structures, we shall now define the first-order span of class $X$ : intuitively, the latter is the collection of all finite-type classes one can obtain from $X$ by repeated applications of the first-order operations; in fact, it is not hard to see that our definition describes the smallest such system.

5.2 Definition. If $X$ is any finite-type class of type $\tau$, then the first-order span of $X$, for short $\operatorname{span}(X)$, is the collection of all finite-type classes $Y$ of the form

$$
Y=\vec{Q} \circ \overrightarrow{k B}\left(\left(\rho_{1} X\right)^{\left\{\dot{x}_{1} \mid \vec{\psi}_{1}\right\}}, \ldots,\left(\rho_{r} X\right)^{\{\dot{x}, \mid \vec{\psi}\}}, E_{1}, \ldots, E_{p}\right)
$$

where $\vec{Q} \circ \vec{k}=Q_{1} k_{1} \ldots Q_{n} k_{n}$ is a prenex function, $B$ a boolean function, $E_{1}, \ldots, E_{p}$ are elementary classes, each $\rho_{i}$ is a name-changer with domain $\tau$, each $\vec{x}_{i}=x_{i}^{1}, \ldots, x_{i}^{z}$ is a sequence of constants, one for each sort of $\rho_{i}(\tau)$, and each $\vec{\psi}_{i}=\psi_{i 1} \ldots \psi_{i z}$ is a sequence where $\psi_{i j}$, of type $\tau_{i j} \supseteq\left\{x_{i}^{j}\right\}$, is a boolean combination of atomic sentences.

Notice that $X \in \operatorname{span}(X)$. We shall try to sketch now the key ideas to represent an equivalence relation $\sim$ as $\equiv_{L}$ for some logic $L$ : one naturally looks for $L$-elementary classes among finite-type classes $X$ such that $\forall Y \in \operatorname{span}(X), Y$ is a union of equivalence classes of $\sim$, since, if $\mathfrak{M} \sim \mathfrak{N}$ then $Y$ cannot separate $\mathfrak{M}$ and $\mathfrak{R}$. One also needs as many as possible such classes $X$, so that whenever not $\mathfrak{M} \sim \mathfrak{N}$ some $X$ actually separates $\mathfrak{M}$ and $\mathfrak{R}$; one then hopes that the system thus obtained is closed under $\wedge$ and $\exists$. We shall see in our main theorem below that this is indeed the case for Robinson equivalence relations satisfying some additional regularity conditions. 
Let us now make precise the above discussion:

5.3 Definition. For $\sim$ a bounded equivalence relation (see $\S 4$ ) on the class of all structures, the hull of $\sim$, for short hull $(\sim)$ is defined by

$$
\begin{aligned}
& X \in \operatorname{hull}(\sim) \text { iff } X \text { is a finite-type class and each } Y \\
& \text { in } \operatorname{span}(X) \text { is a union of equivalence classes of } \sim .
\end{aligned}
$$

Thus, if $X \in$ hull $(\sim), X$ does not separate $\mathfrak{M}, \mathfrak{R}$ if $\mathfrak{M} \uparrow \tau(X) \sim \mathfrak{M} \uparrow \tau(X)$, i.e. it is not the case that $\mathfrak{R} \uparrow \tau(X) \notin X$ and $\mathfrak{M} \uparrow \tau(X) \in X$, and this property is inherited by all $Y \in \operatorname{span}(X)$. Notice that if $\sim$ is finer than $\equiv$, i.e. $\mathfrak{A} \sim \mathscr{B}$ implies $\mathfrak{A} \equiv \mathfrak{B}$, then each elementary class $F$ is automatically in hull( $\sim)$.

5.4 Definition. A bounded equivalence relation $\sim$ is separable iff for each (possibly infinite) type $\tau$ and $\mathfrak{A}, \mathfrak{B} \in \operatorname{Str}(\tau)$, if not $\mathfrak{A} \sim \mathscr{B}$ then there exists $\tau_{0}$ finite $\subseteq \tau$ and $X \in \operatorname{hull}(\sim)$ such that $\mathfrak{A} \uparrow \tau_{0} \in X$ and $\mathfrak{B}_{\uparrow} \tau_{0} \notin X$.

Thus in a separable equivalence relation any two nonequivalent structures can be separated by some $X$ in the hull; notice that if $\sim$ is preserved under reduct, i.e. $\mathfrak{M} \sim \mathfrak{R}$ implies $\mathfrak{M}_{\uparrow} \tau \sim \mathfrak{R}_{\uparrow} \tau$, then from $\mathfrak{A} \uparrow \tau_{0} \in X$ and $\mathfrak{B}_{\uparrow} \tau_{0} \notin X$ with $X \in \operatorname{hull}(\sim)$ one can infer not $\mathfrak{A} \sim \mathfrak{B}$.

We are now ready to state our main representation theorem for equivalence relations on structures:

5.5 TheOREM. (Assuming $\omega$ is the only measurable cardinal): For $\sim$ an arbitrary equivalence relation the following are equivalent:

(a) $\sim$ is bounded, separable, Robinson, preserved under reduct, finer than $\equiv$ and coarser than $\cong$;

(b) $\sim=\equiv_{L}$ for some compact logic $L$ obeying Craig's interpolation, with $\operatorname{Stc}_{L}(\tau)$ having a cardinality $\left|\operatorname{Stc}_{L}(\tau)\right|$ for any $\tau$, and each sentence being of finite type;

(c) $\sim=\equiv_{L}$ for precisely one logic $L$; in addition, $L$ is compact, obeys Craig's interpolation, $\left|\operatorname{Stc}_{L}(\tau)\right|$ exists for any $\tau$, and each sentence in $L$ is of finite type.

The proof is given in the next section.

6. Proof of the representation theorem. The proof that (c) implies (b) is trivial. We now prove that (b) implies (a): assume $\sim=\equiv_{L}$ with $L$ satisfying the hypotheses of (b); then $\sim$ is bounded, as $\left|\operatorname{Stc}_{L} \tau\right|$ exists for all $\tau$; $\sim$ is Robinson, since the assumed properties of $L$ are well known to imply that $L$ satisfies Robinson's consistency theorem; is preserved under reduct, by the expansion axiom satisfied by $L$; $\sim$ is finer than elementary equivalence $\equiv$, since $L \geqslant L_{\omega \omega}$, and is coarser than $\cong$ by the isomorphism axiom satisfied by $L$. We finally prove that $\sim$ is separable: as a matter of fact, let $\mathfrak{A}, \mathfrak{B} \in \operatorname{Str}(\tau)$; assume not $-\mathfrak{A} \sim \mathfrak{B}$, i.e. $\mathfrak{A} \nexists_{L} \mathfrak{B}$; then for some sentence $\varphi \in \operatorname{Stc}_{L}(\tau)$ we have $\mathfrak{A F}_{L} \varphi$ and $\mathfrak{B}_{L} \neg \varphi$; since $\varphi$ is of finite type, then for some finite $\tau_{0} \subseteq \tau, \varphi \in \operatorname{Stc}_{L}\left(\tau_{0}\right)$ and, by the expansion axiom satisfied by $L$, we have that $\mathfrak{A} \backslash \tau_{0} \vDash_{L} \varphi$ and $\mathfrak{B} \backslash \tau_{0} \vDash_{L} \neg \varphi$. Now notice that the finite-type class $X=\left(\bmod _{L} \varphi, \tau_{0}\right)$ is in hull( $\left.\sim\right)$, since for each $Y \in \operatorname{span}(X)$ there exists a sentence $\alpha_{Y} \in \operatorname{Stc}_{L}(\tau(Y))$ such that $Y=\left(\bmod _{L} \alpha_{Y}, \tau(Y)\right)$; this is a consequence of $L$ being closed under the first-order operations. Therefore, $Y$ is a union of equivalence classes 
of $\sim$ and $X(\in$ hull $(\sim))$ indeed separates $\mathfrak{A}$ and $\mathscr{B}$, so that $\sim$ is separable as required. This completes the proof that (b) implies (a).

To prove that (a) implies (c), we first establish the closure properties of hull $(\sim)$ in the following Lemmas 6.1-6.7: we assume throughout that $\sim$ satisfies the conditions in (a).

6.1 Lemma. If $X$ is elementary, then $X \in \operatorname{hull}(\sim)$.

Proof. Otherwise (absurdum hypothesis) $\exists Y \in \operatorname{span}(X)$ which is not a union of equivalence classes of $\sim$, where $Y$ is as in Definition 5.2; notice that each $\left(\rho_{i} X\right)^{\left\{\vec{x}_{i} \mid \psi_{i}\right\}}$ is elementary, hence so is $Y$; therefore $Y$ is a union of equivalence classes of $\equiv$, hence a union of equivalence classes of $\sim$, since $\sim$ is finer than $\equiv$; we have thus a contradiction from the hypothesis that $X \notin \operatorname{hull}(\sim)$.

6.2 LeMma. If $X \in$ hull $(\sim)$ then $X$ is a union of isomorphism classes, i.e. for any $\mathfrak{M}$, $\mathfrak{N}$ with $\mathfrak{M} \cong \mathfrak{R}, \mathfrak{M} \in X$ iff $\mathfrak{R} \in X$.

Proof. $X$ is a union of equivalence classes of $\sim$ and $\sim$ is coarser than $\cong$.

6.3 Lemma. If $X \in \operatorname{hull}(\sim), \rho$ is a name-changer and $Z=\rho(X)$, then $Z \in$ hull $(\sim)$.

Proof. Otherwise (absurdum hypothesis) there exists $Y \in \operatorname{span}(Z)$ which is not a union of equivalence classes of $\sim$, where

$$
Y=\vec{Q} \circ \vec{k} B\left(\left(\rho_{1} Z\right)^{\left\{\dot{x}_{1} \mid \psi_{1}\right\}}, \ldots,\left(\rho_{r} Z\right)^{\left\{\dot{x}_{r} \mid \dot{\psi}_{r}\right\}}, E_{1}, \ldots, E_{p}\right)
$$

as in Definition 5.2. Notice that $\rho_{i} Z=\rho_{i}^{\prime} X$ for a suitable name-changer $\rho_{i}^{\prime}$, so that $Y \in \operatorname{span}(X)$, too, and $Y$ is a union of equivalence classes of $\sim$ as $X \in \operatorname{hull}(\sim)$.

6.4 Lemma. If $X \in \operatorname{hull}(\sim), Z=\neg X$, then $Z \in \operatorname{hull}(\sim)$.

Proof. Otherwise (absurdum hypothesis) $\exists Y \in \operatorname{span}(Z)$ which is not a union of equivalence classes of $\sim$, where

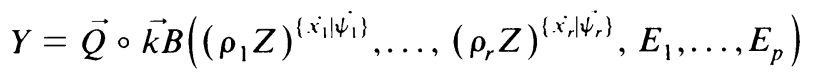

as in Definition 5.2; we have

$$
\left(\rho_{i} Z\right)^{\left\{\dot{x}, \psi_{i}\right\}}=\left(\neg \rho_{i} X\right)^{\{\dot{x}, \mid \vec{\psi}\}}=F_{i} \wedge \neg\left(\left(\rho_{i} X\right)^{\left\{\dot{x}, \mid \psi_{i}\right\}}\right)
$$

with $F_{i}$ elementary, by 5.1(i), so that $Y \in \operatorname{span}(X)$, too, hence $Y$ is a union of equivalence classes of $\sim$ since $X \in \operatorname{hull}(\sim)$.

6.5 Lemma. Assume that $X \in \operatorname{hull}(\sim)$ and $X$ is of finite type $\tau$; let $\vec{y}=y^{1}, \ldots, y^{z}$, respectively, of sort $s_{1}, \ldots, s_{z}$, the latter being precisely the sorts of $\tau$; let $\vec{\varphi}=\varphi_{1}, \ldots, \varphi_{z}$ be a sequence where each $\varphi_{i}$ is a boolean combination of atomic sentences, of type $\tau_{i} \supseteq\left\{y^{i}\right\}$; assume $Z=X^{\{\vec{y} \mid \vec{\varphi}\}}$; then $Z \in \operatorname{hull}(\sim)$.

Proof. Otherwise (absurdum hypothesis) $\exists Y \in \operatorname{span}(Z)$ which is not a union of equivalence classes of $\sim$, where

$$
Y=\vec{Q} \circ \vec{k} B\left(\left(\rho_{1} Z\right)^{\left\{x_{1} \mid \psi_{1}\right\}}, \ldots,\left(\rho_{r} Z\right)^{\left\{\dot{x_{r}} \mid \psi_{r}\right\}}, E_{1}, \ldots, E_{p}\right)
$$


as in Definition 5.2; now we can write

$$
\begin{aligned}
\left(\rho_{i} Z\right)^{\left\{\vec{x}_{i} \mid \vec{\psi}_{i}\right\}} & =\left(\rho_{i}\left(X^{\{\vec{y} \mid \vec{\varphi}\}}\right)\right)^{\left\{\vec{x}_{i} \mid \vec{\psi}_{i}\right\}} \\
& =\left(\left(\rho_{i}^{\prime} X\right)^{\left\{\vec{y}^{\prime} \mid \vec{\varphi}^{\prime}\right\}}\right)^{\left\{\vec{x}_{i} \mid \vec{\psi}_{i}\right\}} \text { for suitable } \vec{y}^{\prime}, \vec{\varphi}^{\prime} \text {, by 5.1(ii) } \\
& =\left(\rho_{i}^{\prime} X\right)^{\left\{\vec{t}_{i} \mid \vec{x}_{i}\right\}} \wedge F_{i} \quad \text { for some } F_{i}, \vec{t}_{i} \vec{\chi}_{i}, \text { as in 5.1(v), }
\end{aligned}
$$

which shows that $Y \in \operatorname{span}(X)$, too, hence $Y$ is a union of equivalence classes of as $X \in \operatorname{hull}(\sim)$.

6.6 Lemma. If $X, Y \in \operatorname{hull}(\sim), Z=X \wedge Y$, then $Z \in \operatorname{hull}(\sim)$.

Proof. Otherwise (absurdum hypothesis) some $E \in \operatorname{span}(Z)$ of the form

$$
E=\vec{Q} \circ \overrightarrow{k B}\left(\left(\rho_{1} Z\right)^{\left\{\vec{x}_{1} \mid \vec{\psi}_{1}\right\}}, \ldots,\left(\rho_{r} Z\right)^{\left\{\vec{x}_{r} \mid \vec{\psi}_{r}\right\}}, E_{1}, \ldots, E_{p}\right)
$$

is not a union of equivalence classes of $\sim$, hence for some $\mathfrak{M}$ and $\mathfrak{N}$ with $\mathfrak{M} \sim \mathfrak{R}$ we have $\mathfrak{M} \in E$ and $\mathfrak{N} \notin E$. Now,

$$
\begin{aligned}
\left(\rho_{i} Z\right)^{\left\{\vec{x}_{i} \mid \vec{\psi}_{i}\right\}} & =\left(\rho_{i}^{\prime} X \wedge \rho_{i}^{\prime \prime} Y\right)^{\left\{\vec{x}_{i} \mid \vec{\psi}_{i}\right\}} \quad \text { for suitable renamings } \rho^{\prime}, \rho^{\prime \prime} \\
& =C_{i} \wedge\left(\rho_{i}^{\prime} X\right)^{\left\{\vec{x}_{i} \mid \vec{\psi}_{i}\right\}^{\prime}} \wedge\left(\rho_{i}^{\prime \prime} Y\right)^{\left\{\vec{x}_{i} \mid \vec{\psi}_{i}\right\}^{\prime \prime}} \text { by } 5.1(\mathrm{iv}) \\
& =C_{i} \wedge K_{i}^{\prime} \wedge K_{i}^{\prime \prime}
\end{aligned}
$$

with $C_{i}, K_{i}^{\prime}$ and $K_{i}^{\prime \prime} \in \operatorname{hull}(\sim)$ by Lemmas $6.1,6.3$ and 6.5 ; therefore $E$ can be written in the form

$$
E=\vec{Q} \circ \overrightarrow{k B} B^{\prime}\left(H_{1}, \ldots, H_{m}\right), \quad H_{1}, \ldots, H_{m} \in \operatorname{hull}(\sim),
$$

for some $m \in \omega$ and $H_{1}, \ldots, H_{m}$ finite-type classes, where $B^{\prime}$ is a new boolean function. Add to $\tau(\mathfrak{M}) m$ new $n$-ary relation symbols $U_{1} \vec{k}, \ldots, U_{m} \vec{k}$ where $\vec{k}=$ $k_{1}, \ldots, k_{n}$ and let $F_{i}$ be given by

$$
F_{i}=\forall \vec{k}\left(\left(U_{i} \vec{k} \wedge H_{i}\right) \vee\left(\neg U_{i} \vec{k} \wedge \neg H_{i}\right)\right), \quad i=1, \ldots, m,
$$

where $U_{i} \vec{k}$ denotes the elementary class determined by $\bmod _{L_{\omega \omega}} U_{i} \vec{k}$.

Notice that $F_{i} \in \operatorname{span}\left(H_{i}\right)$, hence by (1) $F_{i}$ is a union of equivalence classes of $\sim$; if we let

$$
F=F_{1} \wedge \ldots \wedge F_{m}
$$

then $F$, too, is a union of equivalence classes of $\sim$ by the assumed preservation under reduct of $\sim$. Expand $\mathfrak{M}$ to $\mathfrak{M}^{+} \in \operatorname{Str} \tau(F)$ so that

$$
\mathfrak{M}^{+} \in F
$$

the above (definitional) expansion exists by the definition of the $U$ 's. Notice that

$$
\mathfrak{M}^{+} \uparrow \tau(\mathfrak{M}) \in E
$$

hence (1)-(5) are to the effect that

$$
\mathfrak{M}^{+} \in \vec{Q} \circ \overrightarrow{k B^{\prime}}\left(U_{1} \vec{k}, \ldots, U_{m} \vec{k}\right) .
$$


Let $\mathfrak{A}$ be arbitrary, with $\mathfrak{A} \sim \mathfrak{M}^{+}$; then the fact that $\sim$ is finer than $\equiv$, together with (6) implies

$$
\mathfrak{U} \in \vec{Q} \circ \vec{k} B^{\prime}\left(U_{1} \vec{k}, \ldots, U_{m} \vec{k}\right) ;
$$

as a matter of fact, the class in (6) or (7) is elementary. Since $F$ is a union of equivalence classes of $\sim$, by (4) we also have

$$
\mathfrak{A} \in F .
$$

Hence, by (1), (2), (3), (7) and (8) we have

$$
\mathfrak{A} \uparrow \tau(\mathfrak{M}) \in E .
$$

By similarly expanding $\mathfrak{R} \notin E$ via relation symbols $V_{1}, \ldots, V_{m}$ one gets, for any $\mathfrak{B} \sim \mathfrak{R}^{+}$, where $\mathfrak{R}^{+}$is the expanded structure thus obtained,

$$
\mathfrak{B} \mid \tau(\mathfrak{R}) \notin E \text {. }
$$

Hence, there is no $\mathscr{D}$ such that $\mathfrak{D} / \tau\left(\mathfrak{M}^{+}\right) \sim \mathfrak{M}^{+}$and $\mathscr{D} / \tau\left(\mathfrak{N}^{+}\right) \sim \mathfrak{R}^{+}$, since otherwise $\mathfrak{D} \mid \tau(\mathfrak{M})$ is both in $E$ and in $\neg E$ by (9) and (10), which is impossible. Applying now the assumed Robinson property of $\sim$, we obtain that not $\mathfrak{M} \sim \mathfrak{R}$, thus contradicting our assumption about $\mathfrak{M}$ and $\mathfrak{R}$.

6.7 Lemma. If $X \in$ hull( $\sim), h$ is a constant and $Z=\exists h X$, then $Z \in \operatorname{hull}(\sim)$.

Proof. Otherwise (absurdum hypothesis) some $E \in \operatorname{span}(Z)$ of the form

$$
E=\vec{Q} \circ \overrightarrow{k B}\left(\left(\rho_{1} Z\right)^{\left\{\vec{x}_{1} \mid \vec{\psi}_{1}\right\}}, \ldots,\left(\rho_{r} Z\right)^{\left\{\vec{x}_{r} \mid \vec{\psi}_{r}\right\}}, E_{1}, \ldots, E_{p}\right)
$$

is not a union of equivalence classes of $\sim$, hence there are structures $\mathfrak{M}$ and $\mathfrak{R}$ with $\mathfrak{M} \sim \mathfrak{R}$ such that $\mathfrak{M} \in E$ and $\mathfrak{R} \notin E$. Let $s_{t}$ be the sort of $h$, where $1 \leqslant t \leqslant z$ and $s_{1}, \ldots, s_{z}$ are the sorts of $\tau=\tau(X)$. We can write

$$
\left(\rho_{i} Z\right)^{\left\{\vec{x}_{i} \mid \vec{\psi}_{i}\right\}}=\left(\rho_{i} \exists h X\right)^{\left\{\vec{x}_{i} \mid \vec{\psi}_{i}\right\}}=\left(\exists h \hat{\rho}_{i} X\right)^{\left\{\vec{x}_{i} \mid \vec{\psi}_{i}\right\}},
$$

assuming without loss of generality that $h \notin \rho_{i}(\tau)$, where $\hat{\rho}_{i}$ maps $h$ into $h$ and is otherwise equal to $\rho_{i}$.

Therefore we can write

$$
\begin{aligned}
\left(\rho_{i} Z\right)^{\left\{\vec{x}_{1} \mid \vec{\psi}_{i}\right\}} & =\exists h^{\prime}\left(\psi_{i t}^{\prime} \wedge\left(\rho_{i}^{\prime} X\right)^{\left\{\vec{x}^{\prime} \mid \psi^{\prime}\right\}}\right) \text { by } 5.1(\mathrm{iii}) \\
& =\exists h^{\prime} K_{i}
\end{aligned}
$$

for a suitable $K_{i} \in$ hull( $\left.\sim\right)$, by Lemmas $6.3,6.6$ and 6.5. Hence $E$ can be written as

$$
E=\vec{Q} \circ \vec{k} B^{\prime}\left(\exists h^{\prime} K_{1}, \ldots, \exists h^{\prime} K_{r}, E_{1}, \ldots, E_{p}\right), \quad K_{1}, \ldots, K_{r} \in \operatorname{hull}(\sim),
$$

where we assume without loss of generality that $h^{\prime} \neq k_{1}, \ldots, k_{n}$. Add to $\tau(\mathfrak{M}) r$ $n$-ary relation symbols $U_{1} \vec{k}, \ldots, U_{r} \vec{k}$ and let $F_{i}$ be given by (recall 6.6 above for the meaning of $U_{i} \vec{k}$ as a finite-type class):

$$
F_{i}=\forall \vec{k}\left(\left(U_{i} \vec{k} \wedge \exists h^{\prime} K_{i}\right) \vee\left(\neg U_{i} \vec{k} \wedge \forall h^{\prime} \neg K_{i}\right)\right), \quad i=1, \ldots, r,
$$

$$
F_{i}=\forall \vec{k} \exists h^{\prime} \forall h^{\prime \prime}\left(\left(U_{i} \vec{k} \wedge K_{i}\right) \vee\left(\neg U_{i} \vec{k} \wedge \neg K_{i}^{\prime \prime}\right)\right)
$$


where $K_{i}^{\prime \prime}$ is obtained from $K_{i}$ via some renaming which sends $h^{\prime}$ into a new constant $h^{\prime \prime}$; notice that $F_{i} \in \operatorname{span}\left(K_{i}\right)$, hence, by (1), $F_{i}$ is a union of equivalence classes of $\sim$. Let

$$
F=F_{1} \wedge \ldots \wedge F_{r}
$$

we see that $F$, too, is a union of equivalence classes of $\sim$, by $\sim$ being preserved under reduct. Expand $\mathfrak{M}$ to $\mathfrak{M}^{+} \in \operatorname{Str}(\tau(F))$ so that

$$
\mathfrak{M}^{+} \in F \text {. }
$$

Notice that

$$
\mathfrak{M}^{+} \uparrow \tau(\mathfrak{M}) \in E
$$

hence, by (1)-(6) we have

$$
\mathfrak{M}^{+} \in \vec{Q} \circ \overrightarrow{k B}\left(U_{1} \vec{k}, \ldots, U_{r} \vec{k}, E_{1}, \ldots, E_{p}\right) .
$$

Let $\mathfrak{A}$ be an arbitrary structure such that $\mathfrak{A} \sim \mathfrak{M}^{+}$; then by $\sim$ being finer than $\equiv$, (7) implies

$$
\mathfrak{A} \in \vec{Q} \circ \vec{k} B\left(U_{1} \vec{k}, \ldots, U_{r} \vec{k}, E_{1}, \ldots, E_{p}\right)
$$

Since $F$ does not separate any two -equivalent structures, then by (5) we also have $\mathfrak{U} \in F$.

Therefore, by (1)-(4), (8) and (9),

$$
\mathfrak{U} \uparrow \tau(\mathfrak{M}) \in E .
$$

By similarly expanding $\mathfrak{N} \notin E$ to $\mathfrak{N}^{+}$via relation symbols $V_{1}, \ldots, V_{r}$ one gets, for any $\mathfrak{B} \sim \mathfrak{R}^{+}$,

$$
\mathfrak{B} \uparrow(\mathfrak{N}) \notin E \text {. }
$$

Hence, there exists no $\mathfrak{D}$ such that $\mathfrak{D} / \tau\left(\mathfrak{M}^{+}\right) \sim \mathfrak{M}^{+}$and $\mathfrak{D} \mid \tau\left(\mathfrak{N}^{+}\right) \sim \mathfrak{N}^{+}$since otherwise, $\mathfrak{D} \mid \tau(\mathfrak{M}) \in E$ and $\in \neg E$ by (10) and (11), which is impossible. By applying now the assumed Robinson property of $\sim$, we finally have that not $\mathfrak{M} \sim \mathfrak{N}$, a contradiction.

6.8 END OF THE PROOF OF THE THEOREM. Having proved that hull( $\sim)$ is closed under the first-order operations, we define $L=\left(\operatorname{Stc}_{L}, F_{L}\right)$ as follows:

$$
\varphi \in \operatorname{Stc}_{L}(\tau) \text { iff } \varphi \in \operatorname{hull}(\sim) \text { and has type } \tau(\varphi) \subseteq \tau,
$$

and, for $\mathfrak{A} \in \operatorname{Str}(\tau)$,

$(++) \quad \mathfrak{A} F_{L} \varphi \quad$ iff $\varphi \in \operatorname{Stc}_{L}(\tau)$ and $\quad \mathfrak{A} \uparrow \tau(\varphi) \in \varphi$.

We have the following.

Claim 1. $L$ is a logic.

Proof. Directly from the above definition, together with our assumptions about $\sim$ and Lemmas 6.1-6.7; notice that we have not used separability and the special set-theoretical assumption. 
Claim 2. $\equiv_{L}=\sim$.

Proof. First observe that $\equiv_{L}$ is coarser than $\sim$; as a matter of fact, if $\mathfrak{U} \sim \mathscr{B} \in \operatorname{Str}(\tau)$, then by preservation under reduct $\mathfrak{A} \uparrow \tau_{0} \sim \mathscr{B} \uparrow \tau_{0}$ for all finite $\tau_{0} \subseteq \tau$, hence no $X \in$ hull $(\sim)$ can separate $\mathfrak{A}$ and $\mathfrak{B}$, hence $\mathfrak{A} \equiv_{L} \mathfrak{B}$. Therefore, if $\equiv_{L} \neq \sim$ (absurdum hypothesis), then there are $\mathfrak{M}, \mathfrak{R}$ such that $\mathfrak{M} \equiv_{L} \mathfrak{R}$ and not $\mathfrak{M} \sim \mathfrak{R}$; since $\sim$ is separable, there is $\tau_{0}$ finite $\subseteq \tau$ and $Y \in$ hull $(\sim)$ of type $\tau_{0}$ such that $\mathfrak{M} \uparrow \tau_{0} \in Y$ and $\mathfrak{R} \uparrow \tau_{0} \notin Y$. Notice that $Y \in \operatorname{Stc}_{L}\left(\tau_{0}\right)$ and $\mathfrak{M}_{L} Y$ and $\mathfrak{R} \vDash_{L} \neg Y$, whence $\mathfrak{M} \nexists_{L} \mathfrak{R}$, a contradiction.

Claim 3. $L$ is compact, obeys Craig's interpolation, $\left|\operatorname{Stc}_{L} \tau\right|$ exists for any $\tau$, and each sentence in $L$ is of finite type.

Proof. The last two conclusions easily follow from $\sim$ being bounded and from the definition of $L ; L$ is a Robinson logic by Claim 2, since $\sim$ is Robinson; now, from the corollary to the main result in [Mu3], $L$ is compact and obeys Craig's interpolation theorem. Notice that in [Mu3] only relativization to atomic sentences is used, so that the results therein may be safely applied to the logics considered in this paper, where (many-sorted) relativization is to boolean combinations of atomic sentences.

Claim 4. $L$ is the unique logic such that $\equiv_{I}=\sim$.

Proof. Let $L^{\prime \prime}$ be a logic such that $\equiv_{L^{\prime \prime}}=\sim=\equiv_{L}$; then the desired conclusion follows from Theorem 4.2 above.

The proof of Claim 4 concludes the proof of our representation theorem. Q.E.D.

6.9 REMARK. The assumption that $\omega$ is the only measurable cardinal is needed for the proof (of the results in [Mu3] implying) that $(\mathrm{a}) \Rightarrow$ (c) in 5.5 .

As Matt Kaufmann pointed out, in view of a theorem of Friedman [MS1, 4.1] one can delete the hypothesis that each sentence is of finite type in the proof that (b) $\Rightarrow$ (a) in 5.5.

6.10 Remarks. Notice that if one drops the separability assumption on $\sim$, one still gets from $\sim$ a logic $L$ via definitions $(+)$ and $(++)$ in 6.9 above, and $L$ is the strongest logic with $\equiv_{L}$ coarser than $\sim$, and in which every sentence is of finite type. Also notice that the unique logic $L$ given by our representation theorem has many other properties in common with first-order logic, e.g., all the familiar interpolation and definability properties, joint embedding, amalgamation, and $L$ has a Löwenheim number (see [Mu3]); in addition for each sentence $\varphi$ in $L$ there is a smallest finite type $\tau$ such that $\varphi \in \operatorname{Stc}_{L}(\tau)$, so that $L$ automatically satisfies Barwise's "finite occurrence" axiom (see [Ba, pp. 234, 254]). If one restricts attention to logics satisfying such axiom and whose sentences of any given type are equinumerous with a set then (assuming $\omega$ is the only measurable cardinal) Friedman's fourth problem in $[\mathbf{F r}]$ can be given the following equivalent algebraic reformulation:

"Find a bounded separable Robinson equivalence

relation which is preserved under reduct, strictly finer

than $\equiv$ and coarser than $\cong . "$

The author wishes to express his gratitude to Matt Kaufmann. 


\section{REFERENCES}

[Ba] K. J. Barwise, Axioms for abstract model theory, Ann. Math. Logic 7 (1974), 221-265.

[CK] C. C. Chang and H. J. Keisler, Model theory, 2nd ed., North-Holland, Amsterdam, 1977.

[Fe] S. Feferman, Two notes on abstract model theory. I, Fund. Math. 82 (1974), 153-165.

[FI] J. Flum, First-order logic and its extensions, Lecture Notes in Math., vol. 499, Springer-Verlag, Berlin and New York, 1976, pp. 248-310.

[Fr] H. Friedman, One hundred and two problems in mathematical logic, J. Symbolic Logic 40 (1975), 113-129.

[Li] P. Lindström, On extensions of elementary logic, Theoria 35 (1969), 1-11.

[Mo] J. D. Monk, Mathematical logic, Springer-Verlag, Berlin and New York, 1976.

[MS] J. A. Makowsky and S. Shelah, The theorems of Beth and Craig in abstract model theory. I: the abstract setting, Trans. Amer. Math. Soc. 256 (1979), 215-239.

[MS1] _ Positive results in abstract model theory (preprint).

[MSS] J. A. Makowsky, S. Shelah and J. Stavi, $\Delta$-logics and generalized quantifiers, Ann. Math. Logic 10 (1976), 155-192.

[Mu1] D. Mundici, An algebraic result about soft model theoretical equivalence relations with an application to H. Friedman's fourth problem, J. Symbolic Logic 46 (1981), 523-530.

[Mu2] , Robinson's consistency theorem in soft model theory, Trans. Amer. Math. Soc. 263 (1981), $231-241$.

$[\mathrm{Mu3}] \_$_ Compactness $=J E P$ in any logic, Fund. Math. 116 (1982) (to appear).

[Mu4] _ Applications of many-sorted Robinson consistency theorem, Z. Math. Logik Grundlagen Math. 27 (1981), 181-188.

[Mu5] _ Compactness, interpolation and Friedman's third problem (to appear).

[Mu6] __ Embeddings, amalgamation and elementary equivalence (to appear).

[Na] M. E. Nadel, An arbitrary equivalence relation as elementary equivalence in abstract logic, Z. Math. Logik Grundlagen Math. 26 (1980), 103-109.

Loc. Romola N.76, 50060 Donnini, FloRence, Italy 